\title{
DESIGN OF A VHF-BAND RF PHOTOINJECTOR WITH MEGAHERTZ BEAM REPETITION RATE *
}

\author{
J. W. Staples, K. M. Baptiste, J. N. Corlett, S. Kwiatkowski, S. M. Lidia, J. Qiang, F. Sannibale, \\ K. G. Sonnad, S. P. Virostek, R. P. Wells, LBNL, Berkeley, CA 94720 USA
}

\begin{abstract}
New generation accelerator-based X-ray light sources require high quality beams with high average brightness. Normal conducting L- and S-band photoinjectors are limited in repetition rate and $\mathrm{D}-\mathrm{C}$ (photo)injectors are limited in field strength at the cathode. We propose a low frequency normal-conducting cavity, operating at 50 to $100 \mathrm{MHz} \mathrm{CW}$, to provide beam bunches of up to the cavity frequency. The photoinjector uses a re-entrant cavity structure, requiring less than $100 \mathrm{~kW} \mathrm{CW}$, with a peak wall power density less than $10 \mathrm{~W} / \mathrm{cm}^{2}$. The cavity will support a vacuum down to 10 picoTorr, with a load-lock mechanism for easy replacement of photocathodes. The photocathode can be embedded in a magnetic field to provide correlations useful for emittance exchange. Beam dynamics simulations indicate that normalized emittances smaller than $1 \mathrm{~mm}-\mathrm{mrad}$ are possible with gap voltage of $750 \mathrm{kV}$, with fields up to $20 \mathrm{MV} / \mathrm{m}$ at the photocathode, for 1 nanocoulomb charge per bunch after acceleration and emittance compensation. Long-bunch operation (10's of picosecond) is made possible by the low cavity frequency, permitting low bunch current at the $750 \mathrm{kV}$ gap voltage.
\end{abstract}

\section{INTRODUCTION}

We present a high repetition-rate photoinjector, capable of providing pulses up to the cavity frequency itself. In order to achieve the experimental requirements, the requirements for the electron gun include:

- sub $10^{-6} \mathrm{~m}$ normalized beam emittance

- emittance compensation and manipulation techniques that require the presence of solenoidal magnetic fields at the photocathode,

- variable bunch length (from few to several tens of ps) and tunable longitudinal compression factor for trading between space charge effects, photo-cathode requirements and energy spread.

- a charge per bunch of the order of a nC.

- an easy installation and test of different kind of cathodes,

- compatibility between the RF frequencies in the electron gun and RF booster systems with the future main linac.

We are developing at LBNL a room-temperature photoinjector that uses an RF structure operating in the frequency range between 50 to $100 \mathrm{MHz}$ to supply the ini-

\footnotetext{
* This work is supported by the Director, Office of Science, U.S. Dept. of Energy under Contract no. DE-AC02-05CH11231
}

tial beam and acceleration. The lower frequency allows for larger cavities with respect to the $\mathrm{L}$ and $\mathrm{S}$ band case leading to a dramatic reduction of the power density in the RF structure and allowing for $\mathrm{CW}$ operation of the gun. Lower frequencies mean also smaller accelerating gradients respect to the case of L and S band RF structures. Nevertheless, calculations show that this scheme has the potential to operate with very reasonable amount of RF power and achieve gradients and accelerating voltages larger than in a DC gun case.

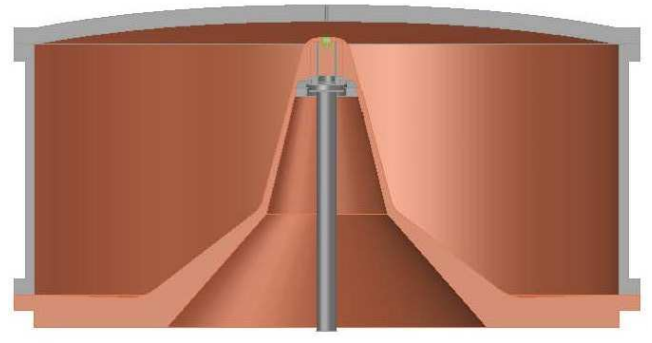

Figure 1: Photoinjector Cavity in Cross Section

The long RF period is compatible with a bunch length of tens of picoseconds, so from the point of view of the beam dynamics the VHF gun behaves as the static field case of DC guns and all the existing results from simulations and calculations for the DC gun case can be used for this VHF RF scheme. The relative large volume of the cavity allows for good vacuum pumping speeds making the structure compatible with operation with photocathodes (GaAs for example). The accelerating gap can be shaped in order to create focusing electric fields, and vacuum load lock techniques can be used for an easy replacement of the photocathodes. The overall size and complexity of the system compares favorably with DC gun based systems and in general, such VHF RF gun has the potential of achieving superior performances respect to DC guns using immediately available and conventional RF technology.

\section{BEAM DYNAMICS}

Beam dynamics simulations have been produced to show feasibility of this RF gun concept with the requirements for high brightness beam production. The APEX (Advanced Photoinjector Experiment) beamline [2] comprises the VHF gun described herein, a UHF (500-750 MHz) bunching cavity, sections of L-band linac structure to accelerate the beam to $50-100 \mathrm{MeV}$, and solenoid magnets for beam transport. Additionally, a solenoid field may be present at the cathode to introduce correlations necessary to 
allow for emittance manipulation techniques downstream of the injector [3]. For beams with $0.5 \mathrm{nC}$ bunch charge, long laser pulses (75ps FWHM) are required to reduce the surface charge density and image forces to allow for extraction without excessive space charge induced emittance growth, with transverse spot size held to $0.5 \mathrm{~mm}$ (RMS) to reduce the thermal emittance contribution. The beam undergoes compression to a final pulse duration of $6.7 \mathrm{ps}$ (RMS) at the entrance to the booster accelerator linacs. Emittance compensation during transport and acceleration to $45 \mathrm{MeV}$ produce a beam with peak current 32A and slice-averaged normalized emittance of $0.35 \mathrm{~mm}$ mrad.

We have conducted studies of the variation of extracted beam quality as the geometry of the cavity is changed and the resonant frequency varies between $65 \mathrm{MHz}$ and 200 $\mathrm{MHz}$. For the small gap size under consideration, the fields remain essentially electrostatic and are unaffected by changes in the resonant frequency over that range. Likewise, the beam dynamics remain essentially unchanged as the frequency varies. We have also examined the effect of changes to the gap geometry (producing more or less transverse electrostatic focusing, deviations from a Pierce electrode geometry, etc.) and have concluded that, for the beam parameters of interest, overall beam quality after compression and emittance compensation is largely independent of the details of the gap electrode geometry. Hence a flat cathode geometry has been adopted in lieu of more complicated shapes.

\section{PHOTOCATHODE}

The characteristics of the photocathode play a fundamental role in defining the performance and the operation mode of a photoinjector. For instance, if one wants to operate an injector with high repetition rate, the limited power available from lasers requires high quantum efficiency cathodes; the thermal emittance of the cathode represents the ultimate performance for the photoinjector in terms of emittance; the mechanism of extraction of the electrons from the cathode determines the shortest bunch length obtainable from the photoinjector; the cathode material can determine photoinjector parameters such as vacuum and compatibility with the RF technique to be used.

In designing our RF-injector we addressed the need for the operation with arbitrary cathodes, and this constraint pushes the requirements for several parameters. For example, very high repetition rates could lead us to use high quantum efficiency GaAs cathodes, and to guarantee a reasonable life time for the cathode, vacuum pressures of the order of $10^{-} 11$ Torr need to be achieved; the RF technology must be compatible with many different cathode materials, pushing towards a preference for normal conductive technology; cathode reactivation and poisoning issues requires the use of a load-lock system; the capability of an easy replacement of the cathode requires a special attention in the mechanical design.

\section{FIELD EMISSION}

Field emission in a photoinjector can lead to degradation of the performance in terms of emittance, to losses of RF power and to cathode lifetime reduction. Simulations can give a qualitative idea about the phenomenon, and comparison with existing results can give a more quantitative feeling on the importance of the phenomenon in our applications. An extensive comparison with existing experimental data from other photoinjectors showed us that for our geometry, for the fabrication techniques that we are considering and with the maximum field in our cavity surface always less than $30 \mathrm{MV} / \mathrm{m}$, field emission should be very limited and should not represent an issue for our design. Depending on the position where the field emission happens, on the time and on the momentum of the emitted electron we can have that the particle is accelerated along the photoinjector (dark current) or can impinge on the injector walls. In particularly severe cases, field emission could create cathode lifetime problems due to electrons back-bombardment.

\section{MULTIPACTORING}

We are analyzing multipacting effects with different geometries using the code MULTIPAC[4]. This is a simulation tool for analyzing electron multipacting in axisymmetric RF structures. Using this tool, we intend to identify points at which multipacting occurs and then alter the geometry slightly at these points until its effect is minimized to a desirable level. Currently scripts have been developed that create input files in the format required by MULTIPAC. MULTIPAC also uses a secondary electron yield (SEY) curve for which we need identify a suitable data set that closely represents the surface properties of the VHF photoinjector.

\section{ION BACK BOMBARDMENT}

To model the effects of ion back bombardment, we have extended the current version of the Impact-T code to include a weighted macroparticle tracking capability for multiple charge states. The electrons are emitted from photocathode and tracked through the RF gun subject to the external accelerating/focusing fields and the self space-charge fields. Within each step of electron motion, instead of using a Monte-Carlo technique for ionization process, we assume that an ion is born at the same location of an electron macroparticle with a weight proportional to the number of real electrons per macroparticle, the neutral gas density, the energy of electron macroparticle, and the ionization cross section. Using a weighted ion macroparticle method, a large number of ion macroparticles are produced during each time step. These ions are further lumped into macroparticle with larger weight by using a grid. Once an ion macroparticle is born, it will be subject to the external accelerating/focusing fields and the space-charge fields 
from the electron bunch. A preliminary result is that the effects of ionization on electrons and the effects of ion on electron beam dynamics are neglected in the simulation since the real number of ions is very small.

\section{THERMAL \& STRUCTURAL ANALYSIS}

A detailed thermal and structural finite element analysis has been carried out for a $65 \mathrm{MHz}$ cavity design, although the final design may be set at a higher frequency. The mechanical concept for the coaxially configured injector cavity consists of a primarily aluminum body with copper plating on the interior surfaces. The inner and outer conductors are comprised of $1 \mathrm{~cm}$ thick cylindrical shells that are 30 $\mathrm{cm}$ and $140 \mathrm{~cm}$ in diameter, respectively. The front and rear covers are made from thicker material and are dished to provide increased stiffness to minimize deflections due to the vacuum loads.

The finite element analysis has been carried out using an ANSYS model. Heat loads were obtained from a Superfish RF analysis and applied to the cavity surfaces of the 2-dimensional, axisymmetric model. The $62 \mathrm{~kW}$ of total power dissipated in the cavity walls will be removed by means of cooling passages on the interior surface of the inner conductor and on the outside of the cavity with a total cooling water flow of approximately $50 \mathrm{gpm}$.

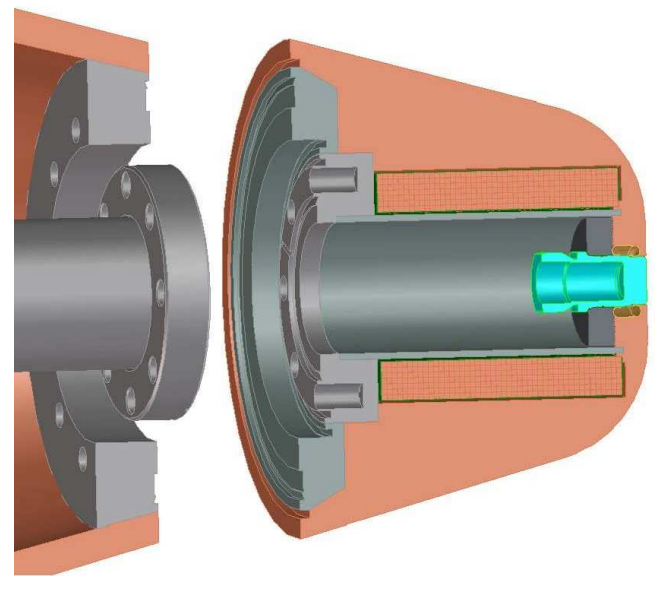

Figure 2: Detachable Nose Assembly

The highest temperature reached is $76 \mathrm{C}$ for $20 \mathrm{C}$ cooling water. With a 2D axisymmetric model, the effect of the discrete, axially running passages is approximated by distributing an effective convection rate evenly on the appropriate surfaces.

The stress and displacement solution is obtained by applying the temperature profile and external atmospheric pressure to an equivalent structural model. The maximum distortion of the cavity is less than $1 \mathrm{~mm}$, and the change in the cavity gap is less than $0.5 \mathrm{~mm}$. Neglecting the effects of stress concentration effects, the peak stress was found to be approximately $27 \mathrm{MPa}$ or about $10 \%$ of the material yield stress.

\section{SOLENOID AND CATHODE MOUNTING}

Provisions for direct adoption of the TTF style cathode load lock and mounting system have been incorporated into the design of a reentrant $88 \mathrm{MHz}$ cavity, shown in Figure 1. In addition, a solenoid has been inserted into the nose of inner conductor surrounding the cathode. The nose subassembly, shown in detail in Figure 2, is separable from the main body of the inner conductor. Concentric conflat gaskets seal both the cathode delivery tube and the inner cavity wall. An annular air space between the cathode delivery tube and the inner cavity wall will be used to supply cooling water to the inner wall surface and solenoid.

\section{RF SOURCES}

For the frequency range of 50 to $100 \mathrm{MHz}$ we will use a CPI 4CW150000E water cooled $150 \mathrm{~kW}$ tetrode in the final amplifier. This is a well-known tube used for many decades for scientific and industrial applications. The anode network of the PA unit will be build as a quarter-wave coaxial structure with a cylindrical anode blocking capacitor. This geometry will create a clean power tube anode impedance spectrum (the closest parasitic resonance is at $3 / 4$ wavelength). The tuning of the anode network will be accomplished with help of a movable short and the impedance matching by adjusting the position of the coaxial line stub inside the anode cavity (conductive coupling of the 4 inch transmission line into the PA unit). For the $50 \mathrm{MHz}$ option the tube would be driven in the common cathode configuration with input impedance of $100 \mathrm{ohm}$ and anode-control grid neutralization circuit. The driver power requirements for this case will be less than $1 \mathrm{~kW}$. $100 \mathrm{MHz}$ option would use power tube in the cathode driven configuration with the DC grounded screen grid. Anode DC voltage would be decreased in this case from $16-19 \mathrm{kV}$ to $12 \mathrm{kV}$. The RF driver power needed in this case would rise to $3 \mathrm{~kW}$.

\section{REFERENCES}

[1] J. Staples, F. Sannibale, S. Virostek, CBP Tech Note, October 2006

[2] S. Lidia, et. al., "Development of a High Brightness VHF Electron Source at LBNL", Proceedings of the 41st Advanced ICFA Beam Dynamics Workshop on Energy Recovery Linacs, Daresbury Laboratory, UK, May 21-25, 2007.

[3] P. Emma, et. al., Phys.Rev.ST Accel.Beams 9:100702,2006.

[4] P. Ylae-Oijala, "Multipacting Analysis and Electromagnetic Field Computation by the Boundary Integral Equation Method in RF Cavities and Waveguides", Ph.D. Dissertation at the Rolf Nevanlinna Institute, University of Helsinki, Finland, (1999) 